Rechtsmedizin $2018 \cdot 28: 85-86$

https://doi.org/10.1007/s00194-018-0232-z

Online publiziert: 28. Februar 2018

(c) Springer Medizin Verlag GmbH, ein Teil von Springer Nature 2018

CrossMark

\author{
B. S. Elger ${ }^{1,3} \cdot$ S. Grabherr ${ }^{1,2}$ \\ ${ }^{1}$ Centre universitaire romand de médecine légale, Université de Genève, Genève, Schweiz \\ ${ }^{2}$ Centre universitaire romand de médecine légale (CURML), Lausanne, Schweiz \\ ${ }^{3}$ Institut für Bio- und Medizinethik, Universität Basel, Basel, Schweiz
}

\title{
Sterbehilfe im internationalen Kontext
}

In den letzten Jahren konnte ein drastischer Wandel in der Gesellschaft festgestellt werden, der insbesondere durch zwei Faktoren herbeigerufen wurde: Unsere Gesellschaft altert anscheinend unaufhörlich, und dank der raschen Entwicklung der medizinischen Versorgung leben immer mehr Menschen mit einer Krankheit, anstatt an ihr zu versterben. Man könnte also annehmen, dass die $\mathrm{Me}$ dizin dabei ist, den Tod zu verdrängen, ja gar zu überwältigen. Obwohl dies zwar auf einem physikalischen Niveau häufig der Fall ist, stellt sich nun aber immer mehr die Frage, ob dies denn überhaupt von den „Überlebenden“ so gewünscht wird. Mehr und mehr Menschen möchten sich selbst ein Limit für ihre Lebenszeit setzen, anstatt auf einen Tod zu warten, der immer weiter hinausgezögert wird. Doch wie das Leben beenden? Ein Suizid ist für viele Menschen eine grausame und gewaltsame Vorstellung. Da dennoch der Wunsch zum Sterben vorhanden ist, wird die Sterbehilfe ein interessantes Thema, das diesen Zwiespalt überbrücken soll. Unausweichlich ist sie somit in den letzten Jahren zu einem Thema in der Rechtsmedizin geworden.

\section{》I Immer mehr Menschen möchten sich selbst ein Limit für ihre Lebenszeit setzen}

Bereits während ihrer 95. Jahrestagung im Herbst 2016 in Heidelberg hat sich die Deutsche Gesellschaft für Rechtsmedizin mit dem Thema „Die alternde Gesellschaft“ beschäftigt. Um es weiter zu vertiefen, widmet sich die Zeitschrift Rechtsmedizin in dieser Sonderausgabe derSter- behilfe. Ziel ist es, die Folgen dieses demografischen Trends auf die Arbeit der Rechtsmediziner $\mathrm{zu}$ beleuchten und $\mathrm{zu}$ definieren, welche Rolle die Rechtsmedizin beim immer wieder diskutierten Thema Sterbehilfe spielt.

Tatsächlich hat in den letzten Jahren eine wachsende Anzahl von Ländern bestimmte Formen von Sterbehilfe und assistiertem Suizid legalisiert. Auch in Deutschland wirft die augenblickliche Entwicklung der Rechtslage wichtige Fragen auf, die ebenso die Rechtsmedizin betreffen. Das vorliegende aktuell zusammengestellte Schwerpunktheft diskutiert v.a. die im Folgenden aufgeführten rechtsmedizinisch bedeutsamen Aspekte.

Aus der Perspektive der Rechtsmedizin ist es zunächst wichtig, Art, Galenik und Nachweis der im Rahmen der Sterbehilfe eingesetzten Substanzen zu kennen. Für die Sterbehilfe und zur Symptomerleichterung in der palliativen Medizin kommen unterschiedliche Medikamente oder Substanzen und unterschiedliche Verabreichungsformen zum Einsatz. Dr. rer. nat. dipl. math. Skopp, Toxikologin aus Heidelberg, stellt diese und ihre Wirkungen in ihrem Beitrag „Sterbehilfe - Medikamente und toxische Substanzen" [5] zusammen.

Im Kontext der Sterbehilfe stellt sich die Frage, welche Aufgaben der Rechtsmedizin bei der Durchführung und Begutachtung von assistiertem Suizid oder Sterbehilfe zukommen und wie sie damit umgehen sollte. In ihrem Beitrag „Assistierter Suizid und Sterbehilfe in einer alternden Gesellschaft. Herausforderung für die Rechtsmedizin" [1] zeigen Prof. Dr. med. B. Elger und Prof. Dr. med. 
S. Grabherr vom Centre universitaire romand de médecine légale der Universitäten Lausanne und Genf, dass das zunehmende Alter der Bevölkerung auch dazu führt, dass immer mehr Personen unter chronischen Krankheiten und erhöhter Gebrechlichkeit leiden. Zusätzlich zu den länderübergreifenden demografischen, ethischen und gutachterlichen Aspekten wird im vorliegenden Schwerpunktheft insbesondere auch auf die aktuelle Rechtslage und Rechtsprechung in Deutschland eingegangen.

Die Autoren J. Laux, J. Nahrmann und M. Parzeller vom Institut für Rechtsmedizin am Klinikum der Johann Wolfgang Goethe-Universität, Frankfurt am Main, geben einen Überblick zu ärztlicher Sterbehilfe in der strafrechtlichen Praxis [2].

Ein wichtiger Urteilskommentar (Das „Sterbehilfe“-Urteil des BGH vom 25.06.2010, 2 StR 454/09 und die These der Gleichsetzung von Tun und Unterlassen, [3]) wurde von Frau Prof. Dr. jur. $R$. Rissing van Saan beigetragen. Sie ist ehemalige Vorsitzende Richterin des Senats des Bundesgerichtshofs (BGH), der das von ihr kommentierte Urteil verfasst hat. Der Beitrag nimmt zum deutschen Patientenverfügungsgesetz Stellung und diskutiert ebenfalls die bis dahin gängige Rechtsprechung des BGH in Zivil- und Strafrecht zu den Voraussetzungen der in Deutschland rechtmäßigen „passiven Sterbehilfe“.

Ein hochaktuelles Urteil aus dem Jahr 2017 wird von Dr. jur. utr. C. Schütz und Dr. med. T. Sitte in ihrem Beitrag „Palliativversorgung statt Lebensverkürzung: BVerwG-Urteil mangelt es im Tatsächlichen" [4] kommentiert. Die Autoren gehen auf die (verfassungs)rechtliche Bewertung und Erörterung der Konsequenzen des Urteils des Bundesverwaltungsgerichts vom 07.03.2017 (Az. 3 C 19/15) und vom 02.03.2017 (Az. 3C 19.15) für die Zukunft ein. Dabei überprüfen sie auch die Urteilsgründe am Maßstab der aktuellen Grundrechtsdogmatik, insbesondere des Verhältnismäßigkeitsgrundsatzes.

Wir hoffen, dass mit diesem Schwerpunktheft der Zeitschrift Rechtsmedizin die Diskussion über ethische und rechtliche Aspekte der Sterbehilfe und des assistierten Suizids angeregt werden kann. Die vorliegenden Beiträge sollen den Lesern bewusst machen, wie wichtig dieses Thema und das Hintergrundwissen dazu für die Rechtsmedizin ist. Immerhin kommt ihr durch die Beantwortung dieser Fragen eine bedeutsame Rolle für die Stärkung von Rechtsstaatlichkeit und Rechtssicherheit zu. Aus diesem Grund stellt sich dieses Heft den Herausforderungen der Zukunft, indem es das Thema und die dabei aufkommenden Fragen aus verschiedenen Perspektiven der Toxikologie, der Palliativmedizin, der Ethik, des Rechts und der Rechtsprechung beleuchtet.

Prof. Dr. Bernice Elger

Prof. Dr. Silke Grabherr

\section{Korrespondenzadresse}

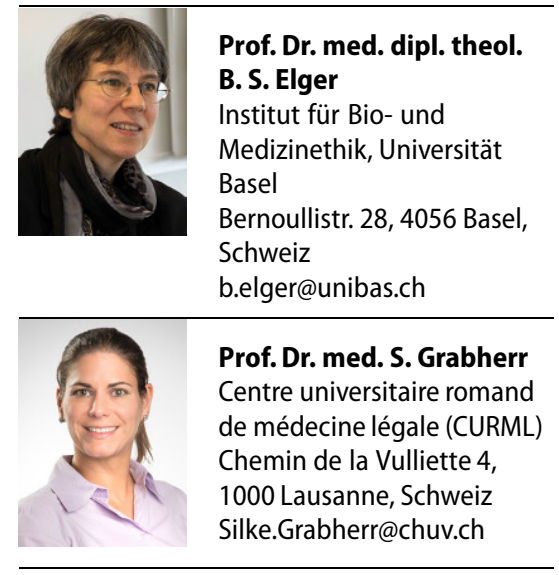

Interessenkonflikt. B.S. Elger und S. Grabherr geben an, dass kein Interessenkonflikt besteht.

\section{Literatur}

1. Elger BS, Grabherr S (2017) Assistierter Suizid und Sterbehilfe in einer alternden Gesellschaft. Herausforderung für die Rechtsmedizin. https://doi. org/10.1007/s00194-017-0205-7. Zugegriffen: 10. Nov. 2017

2. Laux J, Nahrmann J, Parzeller M (2018) Ärztliche Sterbehilfe in der strafrechtlichen Praxis - ein Überblick. Rechtsmedizin 28. https://doi.org/10. 1007/s00194-018-0230-1

3. Rissing-Van Saan R (2017) Das "Sterbehilfe“-Urteil des BGH vom 25.06.2010, 2 StR 454/09 und die These der Gleichsetzung von Tun und Unterlassen. Rechtsmedizin, https://doi.org/10.1007/s00194017-0211-9.Zugegriffen:6. Okt. 2017

4. Schütz C, Sitte T (2018) Palliativversorgung statt Lebensverkürzung. BVerwG-Urteil mangelt es im Tatsächlichen. Rechtsmedizin 28. https://doi.org/ 10.1007/s00194-018-0233-y

5. Skopp G (2017) Sterbehilfe - Medikamente und toxische Substanzen. Rechtsmedizin. https://doi. org/10.1007/s00194-017-0207-5.Zugegriffen: 6 . Okt. 2017 